\title{
金属锰配合物催化的氢化反应中的配体效应一一机理与应用
}

\author{
何艳梅范青华*
}

(中国科学院化学研究所 北京 100190)

\section{Ligand Effect in Maganese-Catalyzed Hydrogenation: Mechanism and Application Studies}

\author{
He, Yanmei Fan, Qinghua* \\ (Institute of Chemistry, Chinese Academy of Sciences, Beijing 100190)
}

过渡金属催化不饱和有机化合物的氢化反应是有 机合成当中最为重要并广泛研究的反应之一. 因其具有 高原子经济性及操作简单方便的特点, 符合绿色可持续 发展化学的要求, 所以一直位于有机化学研究的前沿, 并在工业生产中得到实际应用. 目前, 该反应通常需要 使用铑、钓、铱和钯等贵金属催化剂才能高效实现. 由 于这些贵金属面临着资源枯竭、价格昂贵及重金属残留 等问题，所以亟待发展储量丰富、经济实用且环境友好 的新型廉价金属催化剂.

锰元素是全球储量第三的过渡金属元素, 金属锰配 合物催化的有机反应近年来受到科研人员的广泛关注. 2016 年, 第一例锰金属催化的氢化反应由 Beller 等 ${ }^{[1]}$ 率 先发表. 他们使用 PNP 钳形三齿配体与 $\mathrm{Mn}(\mathrm{CO})_{5} \mathrm{Br}$ 形 成的金属配合物 $\mathbf{1}$ (图 1), 实现了一系列腈、醛和酮类化 合物的高效氢化. 随后, 通过使用类似的 PNP 和 NNP 三齿配体, Beller ${ }^{[2,3]}$ 、Milstein ${ }^{[4]}$ 、Rueping ${ }^{[5]}$ 和 Kempe $^{[6]}$ 等相继报道了酯、酰胺和亚胺的高效氢化. 与此同时, 手性三齿配体的应用也将锰配合物引入到更具挑战性 的不对称催化氢化领域. 最近, Clarke ${ }^{[7]} 、$ Beller ${ }^{[8]}$ 和丁奎 岭课题组 ${ }^{[9]}$ 相继报道了锰-手性三齿配体配合物催化酮 和酯类化合物的不对称氢化. 值得关注的是, 丁奎岭等 发展的催化剂 10(图 1)最为高效, 转化数可达 9800, 底 物适用性广, 基团耐受性强, 获得手性醇的对映体过量 也最高 $(85 \% \sim 98 \% e e)$.

在已报道的锰催化氢化反应中, 反应机理的研究相 对较少 ${ }^{[7]}$. 最近, 清华大学刘强课题组联合重庆大学蓝 宇课题组 ${ }^{[10]}$ 将实验与理论计算相结合, 通过深入系统 的构-效关系研究, 揭示了锰催化氢化反应中配体效应
的本质.

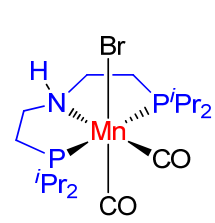

1

2016, Beller

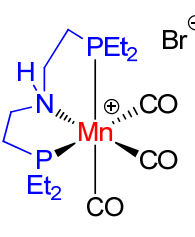

2

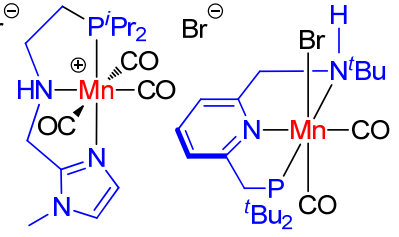

3
2016, Beller 2017, Beller 2018, Milstein

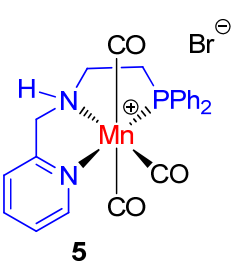

2018, Rueping

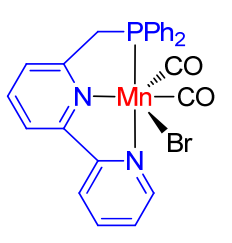

6

2019, Milstein

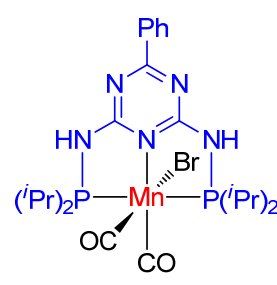

7 2019, Kempe

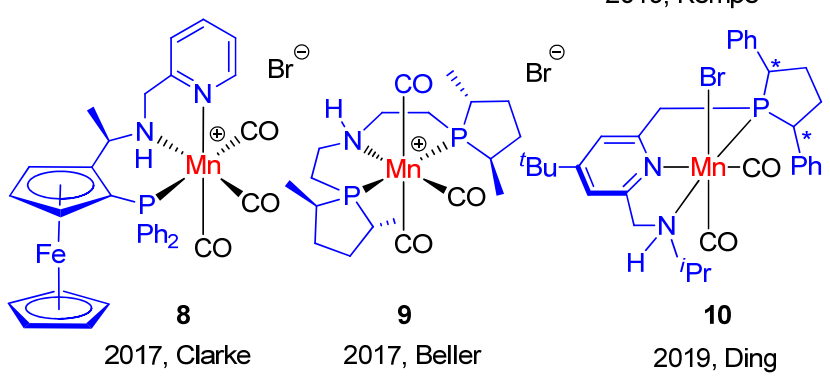

图 1 锰催化氢化中的代表性催化剂

Figure 1 Representative manganese complexes in catalytic hydrogenation

首先，通过比较不同结构 NNP 和 PNP 钳形锰配合 物在酰胺氢化反应中截然不同的表现及其锰配合物中 $\mathrm{CO}$ 红外振动吸收频率, 结合密度泛函理论(DFT)计算

* Corresponding author. E-mail: fanqh@iccas.ac.cn. published online November 9, 2019. 
其空间、电子效应对过渡态能垒和负氢解离能的影响, 证明了 NNP 钳形锰配合物相比于 PNP 钳形锰配合物给 电子能力更强、空间位阻更小. 因此, NNP 钳形锰配合 物在惰性底物的氢化中表现出更高的反应活性.

随后，他们首次将这些三齿钳形配体应用于锰催化 含氮芳杂环化合物的氢化反应中(图 2). 发现 NNP 钳形 锰配合物同样表现出比 PNP 钳形锰配合物更高的反应 活性, 并实现了 32 种不同底物的高效高选择性氢化. 机 理研究表明, 模型底物喹啉的氢化经历了 1,2-加氢-异 构化-亚胺氢化的串联反应途径(图 3), 不同配体在反应 决速步 1,2-加氢过程中存在反应活性的明显差异.

总之, 刘强课题组和蓝宇课题组将实验与理论计算 相结合, 阐明了金属锰配合物催化氢化反应中配体效应 的本质, 从理论上解释了 NNP 钳形锰配合物相比于 PNP 钳形锰配合物具有更高催化活性的原因; 并基于该
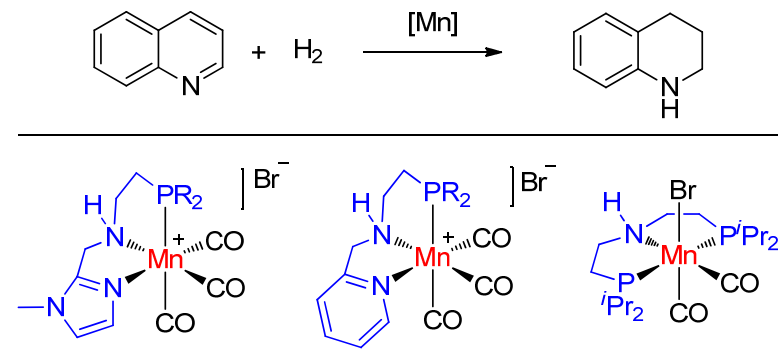

$\mathrm{R}={ }^{i} \mathrm{Pr}, \mathrm{Ph}: 99 \%$ yield

$\mathrm{R}={ }^{i} \mathrm{Pr}, \mathrm{Ph}: 99 \%$ yield

$5 \%$ yield

图 2 锰催化喹啉氢化中的配体效应

Figure 2 Ligand effects in Mn-catalyzed hydrogenation of quinoline

机理，发展了首例锰催化的含氮芳杂环的高效氢化，将 对未来新型多齿配体和金属锰催化剂的设计合成提供 指导作用.

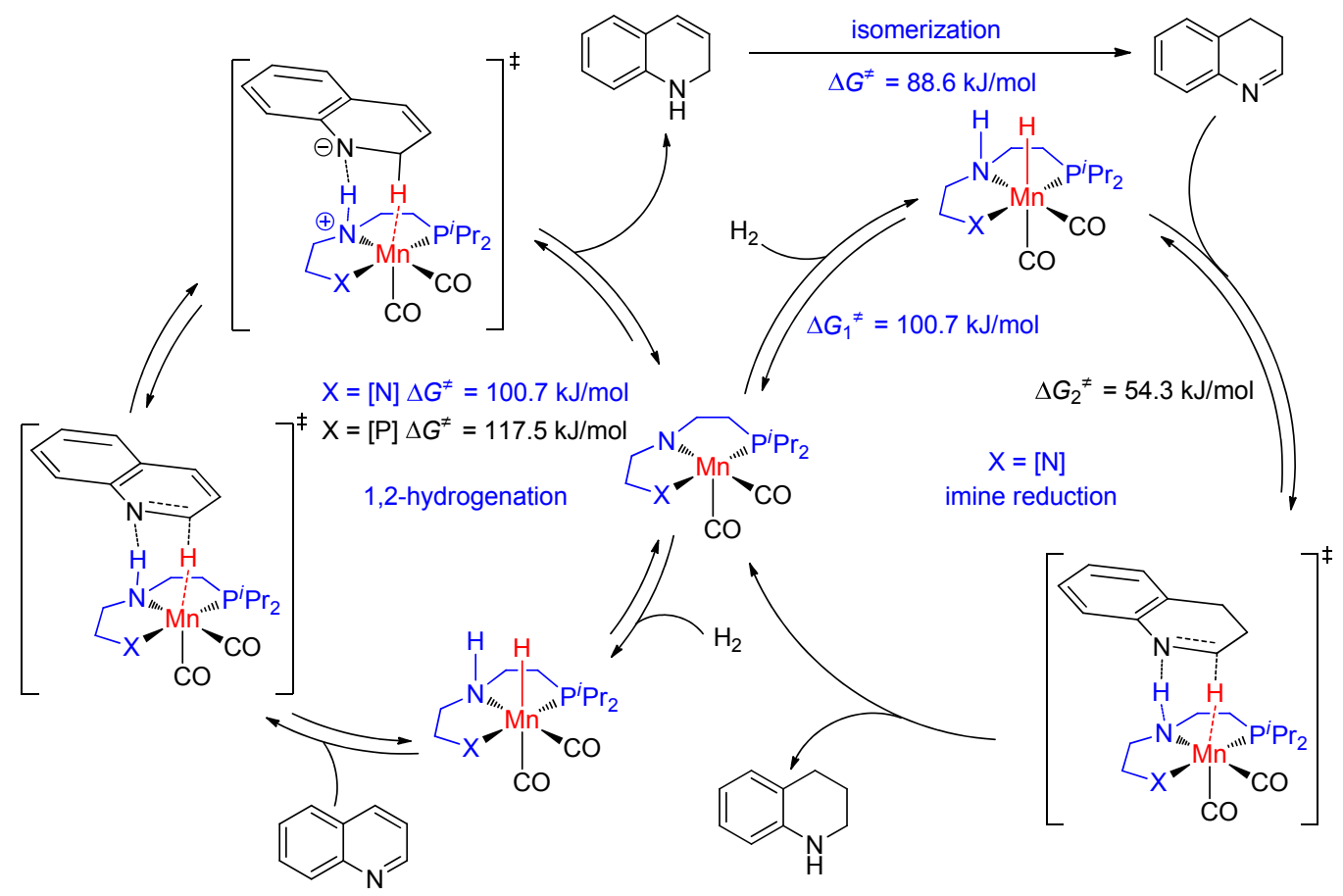

图 3 锰催化喹啉氢化的机理

Figure 3 Proposed mechanism for the Mn-catalyzed hydrogenation of quinoline

\section{References}

[1] Elangovan, S.; Topf, C.; Fischer, S.; Jiao, H.; Spannenberg, A.; Baumann, W.; Ludwig, R.; Junge, K.; Beller, M. J. Am. Chem. Soc. 2016, 138, 8809 .

[2] Elangovan, S.; Garbe, M.; Jiao, H.; Spannenberg, A.; Junge, K.; Beller, M. Angew. Chem., Int. Ed. 2016, 55, 15364.

[3] Papa, V.; Cabrero-Antonino, J. R.; Alberico, E.; Spanneberg, A.; Junge, K.; Junge, H.; Beller, M. Chem. Sci. 2017, 8, 3576.

[4] Kumar, A.; Janes, T.; Espinosa-Jalapa, N. A.; Milstein, D. Angew. Chem., Int. Ed. 2018, 57, 12076.

[5] Zubar, V.; Lebedev, Y.; Azofra, L. M.; Cavallo, L.; El-Sepelgy, O.;
Rueping, M. Angew. Chem., Int. Ed. 2018, 57, 13439.

[6] Freitag, F.; Irrgang, T.; Kempe, R. J. Am. Chem. Soc. 2019, 141, 11677.

[7] Widegren, M. B.; Harkness, G. J.; Slawin, A. M. Z.; Cordes, D. B.; Clarke, M. L. Angew. Chem., Int. Ed. 2017, 56, 5825.

[8] Garbe, M.; Junge, K.; Walker, S.; Wei, Z.; Jiao, H.; Spannenberg, A.; Bachmann, S.; Scalone, M.; Beller, M. Angew. Chem., Int. Ed. 2017, 56, 11237.

[9] Zhang, L.; Tang, Y.; Han, Z.; Ding, K. Angew. Chem., Int. Ed. 2019, 58, 4973.

[10] Wang, Y.; Zhu, L.; Shao, Z.; Li, G.; Lan, Y.; Liu, Q. J. Am. Chem. Soc. 2019, 141, 17337.

(Zhao, C.) 